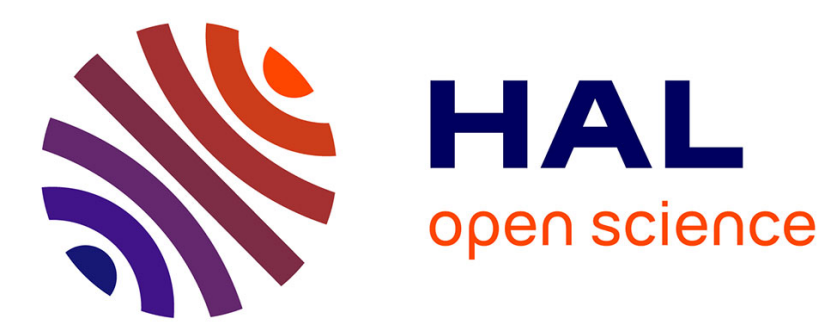

\title{
Un cas d'application des équations de Landau-Ginzburg dépendant du temps.L'anisotropie d'impédance de surface dans les supraconducteurs de type II sales
}

\author{
P. Monceau, G. Waysand
}

\section{- To cite this version:}

P. Monceau, G. Waysand. Un cas d'application des équations de Landau-Ginzburg dépendant du temps.L'anisotropie d'impédance de surface dans les supraconducteurs de type II sales. Revue de Physique Appliquée, 1973, 8 (4), pp.413-415. 10.1051/rphysap:0197300804041300 . jpa-00243706

HAL Id: jpa-00243706

https://hal.science/jpa-00243706

Submitted on 1 Jan 1973

HAL is a multi-disciplinary open access archive for the deposit and dissemination of scientific research documents, whether they are published or not. The documents may come from teaching and research institutions in France or abroad, or from public or private research centers.
L'archive ouverte pluridisciplinaire HAL, est destinée au dépôt et à la diffusion de documents scientifiques de niveau recherche, publiés ou non, émanant des établissements d'enseignement et de recherche français ou étrangers, des laboratoires publics ou privés. 


\title{
UN CAS D'APPLICATION DES ÉQUATIONS DE LANDAU-GINZBURG DÉPENDANT DU TEMPS. L'ANISOTROPIE D'IMPÉDANCE DE SURFACE DANS LES SUPRACONDUCTEURS DE TYPE II SALES
}

\author{
P. MONCEAU \\ Centre de Recherches sur les Très Basses Températures, Cedex 166-38042 Grenoble, France \\ G. WAYSAND \\ Physique des Solides, Université Paris Sud, 91405 Orsay, France
}

\begin{abstract}
Résumé. - La dispersion des résultats expérimentaux concernant l'anisotropie d'impédance de surface dans les supraconducteurs de type II sales est attribuée à l'existence de vortex inclinés dans le tégument de surface.
\end{abstract}

\begin{abstract}
The dispersion of experimental results concerning the anisotropy of surface impedance in dirty type II superconductors is attributed to the existence of tilted vortices in the surface sheath.
\end{abstract}

Les équations de Ginzburg-Landau dépendantes du temps n'ont d'expression vraiment simple que pour la région sans bande interdite des supraconducteurs avec impuretés magnétiques. C'est malheureusement une situation difficile à réaliser. C'est pourquoi les expériences sur la cinétique des supraconducteurs ont été faites sur les matériaux de type II sales et ont, avant tout traitement théorique, mis en évidence l'anisotropie de l'impédance de surface [1].

Soit un supraconducteur de type II ayant une surface plane et plongé dans un champ magnétique $\mathbf{H}$ continu parallèle à la surface. Lorsqu'on irradie cette surface par une onde hyperfréquence plane, parallèle à la surface de l'échantillon, on constate que la puissance hyperfréquence absorbée par l'échantillon est plus forte lorsque la composante électrique $\mathbf{E}_{\boldsymbol{\omega}}$ de l'onde hyperfréquence est perpendiculaire à $\mathbf{H}$ que lorsqu'elle lui est parallèle. Il y a anisotropie de l'impédance de surface : elle est plus élevée dans l'orientation transverse $\left(\mathbf{E}_{\omega} \perp \mathbf{H}\right)$ que dans l'orientation longitudinale $\left(\mathbf{E}_{\omega} / / \mathbf{H}\right)$ (Fig. 1).

Par analogie avec l'écoulement de flux («flux flow ») il est facile de comprendre que lorsque $\mathbf{E}_{\omega} \perp \mathbf{H}$ il y a couplage entre le courant d'écrantage et le champ électrique. On a une véritable oscillation forcée du tégument de surface qui est la réponse du supraconducteur perturbé par le champ hyperfréquence. Cette oscillation n'est en fait, comme l'ont montré Caroli et Maki [2], qu'un mode collectif de fluctuation du paramètre d'ordre. La perturbation étant vectorielle $\left(\mathbf{E}_{\omega}\right)$, la réponse l'est aussi : dans le cas où le champ magnétique est perpendiculaire à l'échantillon, on a affaire
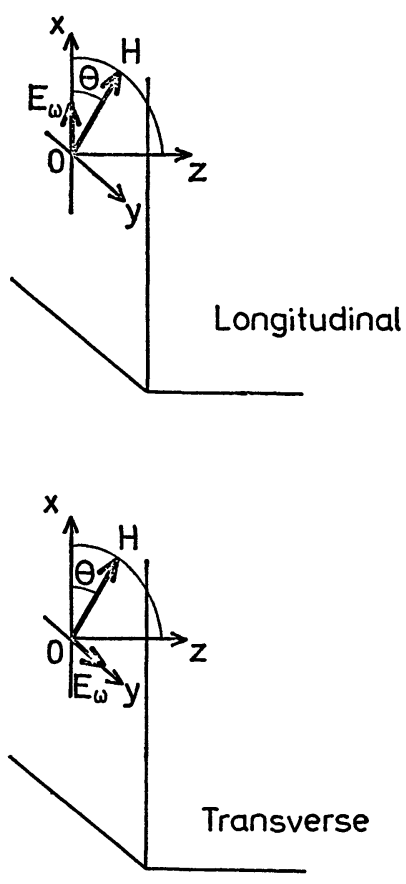

FIG. 1.

à un déplacement oscillatoire de l'ensemble du réseau de vortex. Le caractère remarquable des modes collectifs de fluctuation est qu'ils existent même si la perturbation initiale est très petite.

Le travail de Caroli et Maki devait être corrigé un peu plus tard par Thompson [3]. Dans le cas d'un champ magnétique parallèle à la surface, à la différence de Caroli et Maki, Thompson prédit une aniso- 
tropie $(*)$ d'impédance indépendante de la température et égale à $0,586 \mathrm{si}$ on est suffisamment loin de $T_{\mathrm{c}}$ (il faut $\hbar \omega<\pi\left(T-T_{\mathrm{c}}\right)$ où $\omega$ désigne la fréquence incidente, $T$ la température à laquelle est faite la mesure) et si on a affaire à un supraconducteur de type II prononcé $(\kappa \gg 1)$.

La figure 2 montre une partie des résultats expérimentaux obtenus ces derniers temps par Baixeras et Bodin [4] ainsi que par nous-mêmes. Les matériaux employés répondent aux critères de Thompson, les mesures sont faites sur trois montages différents tant par leur fréquence $(600 \mathrm{MHz}, 2,46 \mathrm{GHz}, 9,45 \mathrm{GHz})$ que par le résonateur utilisé. Sont aussi portés sur la figure les résultats obtenus antérieurement par Fischer et Maki [5] à partir de mesures à $9,5 \mathrm{GHz}$.

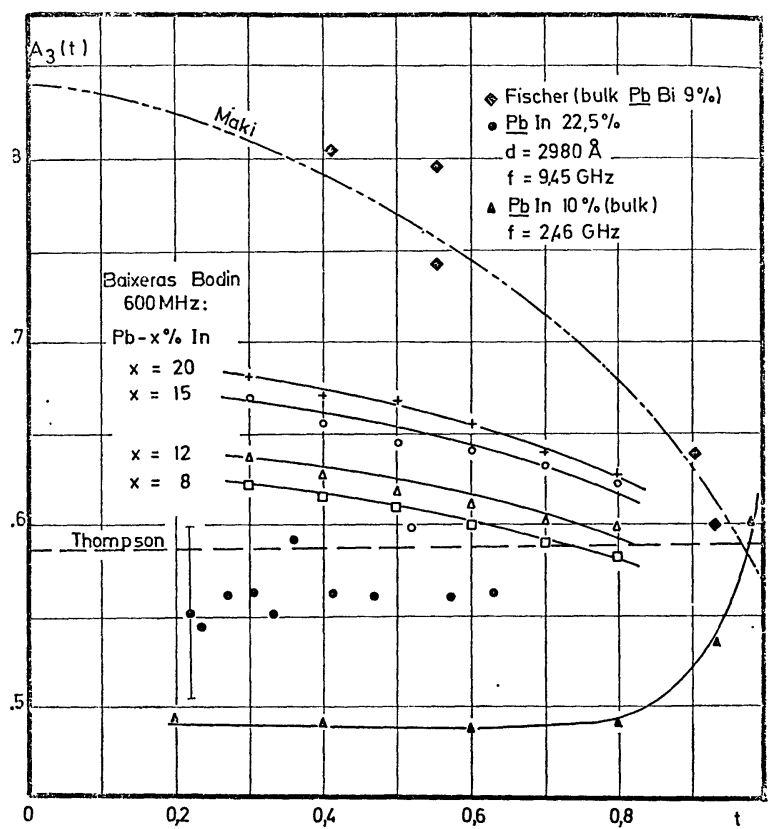

FIG. 2.

Si on exclut la région proche de $T_{\mathrm{c}}$ où les calculs de perturbation ne s'appliquent plus, on voit que l'ensemble des valeurs obtenues pour l'anisotropie en fonction de la température couvrent à peu près tout l'espace compris entre les prédictions de Caroli-Maki et celles de Thompson. Pour les mesures que nous avons effectuées à $9,45 \mathrm{GHz}$ la constance de l'anisotropie est approximativement vérifiée mais de toute façon la valeur obtenue ne correspond jamais à celle de Thompson. Les alliages utilisés étant à base de $\mathrm{Pb}$, on a affaire à des supraconducteurs à couplage fort pour lesquels les calculs sont évidemment moins bien vérifiés.

$\left(^{*}\right)$ Rappelons qu'on opère au voisinage du champ critique pour rester dans le domaine de validité des équations de Ginzburg-Landau. L'anisotropie est alors définie comme le rapport des pentes au voisinage dı champ critique des courbes de transition dans les orientations transverses et longitudinales.
Cet argument assez traditionnel pour expliquer des désaccords entre théorie et expérience dans les supraconducteurs de type II ne semble pas avoir sa place dans le cas présent. Des mesures effectuées à $600 \mathrm{MHz}$ par Baixeras et Bodin sur des alliages PbIn de diverses concentrations, il résulte en effet que ce sont les alliages les moins concentrés en indium qui suivent le mieux les prédictions de Thompson. La discussion des conditions réelles des mesures d'anisotropie nous semble donc plus fructueuse que le recours à un argument très général. En effet, aucun des montages réalisés jusqu'à présent ne permet de réaliser vraiment un parallélisme parfait entre le champ $H$ et la surface de l'échantillon dans les deux orientations transverse et longitudinale. Or, Monceau a montré [6] que dès qu'on incline le champ magnétique sur la surface de l'échantillon, l'absorption hyperfréquence augmentait très rapidement. A partir de ces observations, Maki a développé deux calculs différents de dépendance angulaire [7], [8] qui fournissent le même résultat : il existe un angle $\theta_{c}$ entre le champ et la surface pour lequel la pente de l'absorption au voisinage du champ critique n'a plus que la moitié de sa valeur en champ strictement parallèle $\left(\theta=0^{\circ}\right)$. Cet angle est défini par

$$
\sin \theta_{\mathrm{c}}=\frac{\omega}{4 e D H}
$$

où $D=\frac{1}{3} v_{\mathrm{F}} l$ est le coefficient de diffusion des électrons et $H \sim H_{\mathrm{c}_{3}}$. Appliquée aux alliages PbIn $20 \%$, cette formule fournit des angles de l'ordre de $1^{0}$ à $10 \mathrm{GHz}$ et $4,2 \mathrm{~K}$ et quelques centièmes de degré à $600 \mathrm{MHz}$ et $4,2 \mathrm{~K}$. Les expériences de Monceau (Fig. 3) vérifient qualitativement cet ordre de grandeur. $\theta_{\mathrm{c}}$ permet donc d'estimer la précision angulaire nécessaire pour que les mesures d'anisotropie aient du

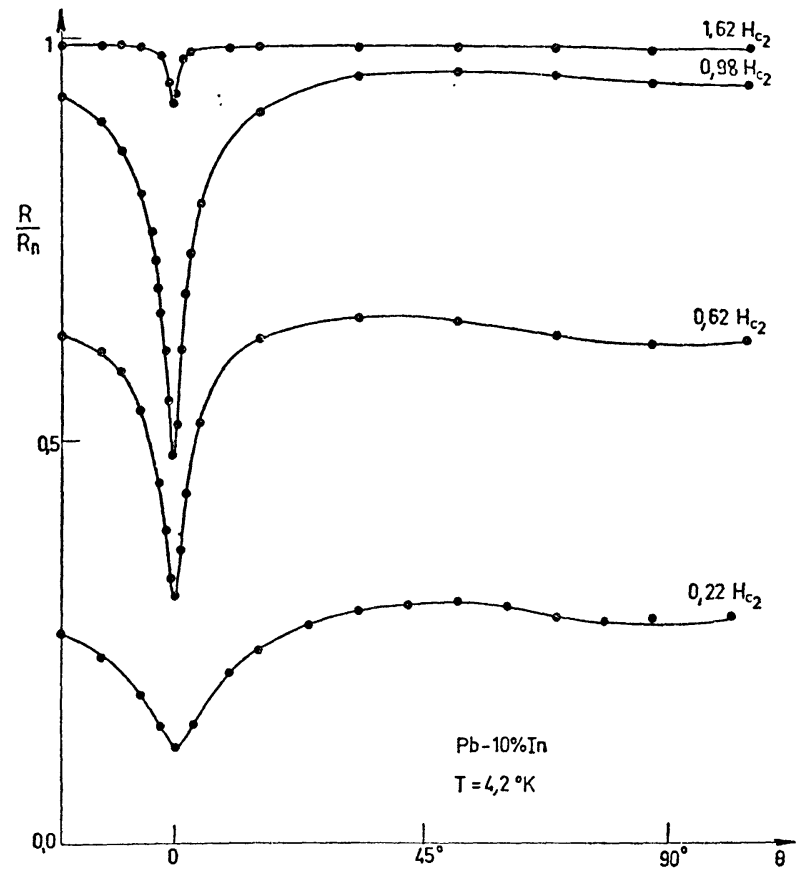

FIG. 3. 
sens. Même dans le cas de mesures à $10 \mathrm{GHz}$ on voit que la condition est draconienne : le plan de l'échantillon et le plan de rotation du champ magnétique doivent être parallèles à une fraction de degré près. L'impédance de surface a donc une dépendance angulaire bien plus aiguë que celle du champ critique puisqu'il faut un angle d'environ $13^{\circ}$ pour que ce dernier décroisse de la moitié de la quantité $\left(H_{\mathrm{c}_{3}}-H_{\mathrm{c}_{2}}\right)$ [9], [10]. Ce n'est pas l'anisotropie de l'impédance en champ parallèle que l'on mesure mais l'anisotropie de deux situations où le champ est incliné de manière différente.

Ce qui explique la rapide décroissance de l'impédance avec l'inclinaison, c'est la présence dans le tégument, dès que le champ n'est plus parallèle, de vortex inclinés. Dans le cœur de ces vortex, la conductivité est évidemment bien moins grande que dans le reste du tégument : l'absorption hyperfréquence croît avec l'angle d'inclinaison. En fait, cette croissance n'est pas monotone, elle est compliquée par toute une série d'interférences du réseau de vortex avec le tégument [11], [12].

La figure 4 montre un exemple de dépendance angulaire observée avec une résolution de $0,5^{\circ}$. La non-monotonie de la décroissance rend impossible

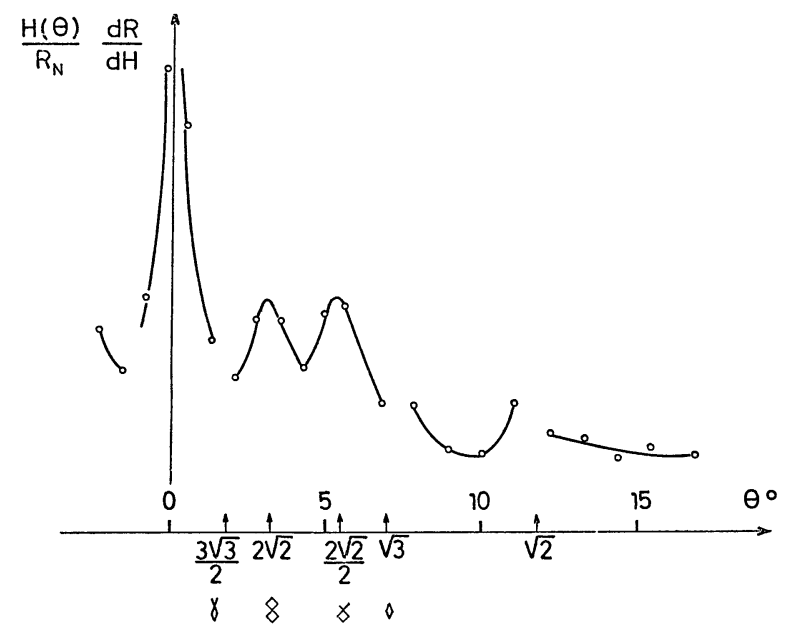

FIG. 4.

toute correction des valeurs expérimentales de l'anisotropie. Un calcul complet doit être entrepris, qui prenne explicitement en compte les extréma. Ce calcul est sans doute plus aisé pour la situation longitudinale
(Fig. 5a). Dans ce cas (inclinaison par rapport à l'orientation longitudinale) le vecteur $\mathbf{e}_{\omega} \wedge \mathbf{H}$ qui définit la direction de fluctuation reste constamment dans le plan de la surface de l'échantillon. Aucune fluctuation du tégument proprement dit n'est excitée dans ce cas, seuls les vortex inclinés sont concernés. Quand on tourne le champ $H$ de 0 à $\pi / 2$ sur la surface, ce sont seulement les fluctuations des vortex qui varient depuis 0 jusqu'à leur valeur calculée par Caroli Maki Thompson à $H_{\mathrm{c}_{2}}$. Au contraire (Fig. 5b) dans le cas où le champ est incliné d'un angle par rapport à l'orientation longitudinale le vecteur $\mathbf{e}_{\omega} \wedge \mathbf{H}$ fait un angle variable de $\pi / 2-\theta$ avec la surface. Les vortex inclinés et le tégument interagissent car ils sont tous les deux soumis à des fluctuations à cause de l'existence d'une composante perpendiculaire à la surface du vecteur $\mathbf{e}_{\omega} \wedge \mathbf{H}$. Cet effet reste entièrement à calculer.

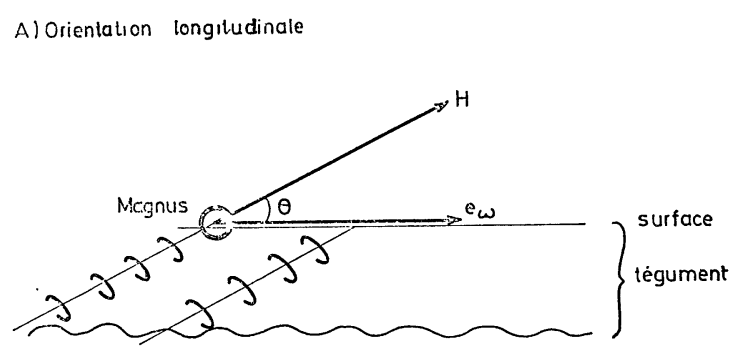

B) Orientution transverse

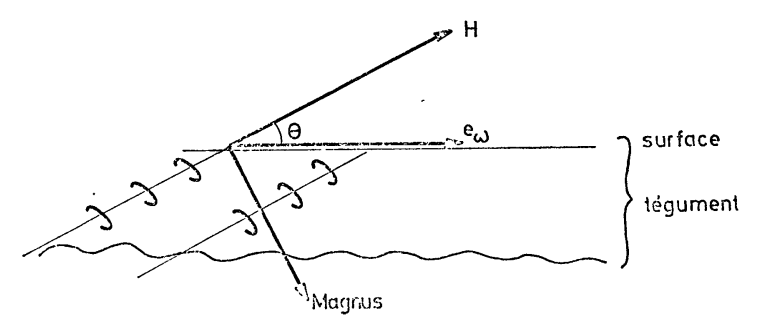

FIG. 5.

En conclusion, l'anisotropie d'impédance de surface qui est un effet spectaculaire ne permet de vérification satisfaisante des solutions proposées pour les équations de Ginzburg-Landau dépendantes du temps qu'au prix d'une extension de ces solutions à des angles non nuls entre champ magnétique continu et surface de l'échantillon.

\section{Bibliographie}

[1] Cardona, M., Fischer, G. and Rosenblum, B., Phys. Rev. Lett. 12 (1964) 101.

[2] Caroli, C. et Maki, K., Phys. Rev. 159 (1967) 306.

[3] Thompson, R. S., Phys. Rev. B 3 (1971) 1617.

[4] Baixeras, J. et Bodin, C., J. Physique 33 (1972) 407.

[5] Fischer, G. et MaKi, K., Phys. Rev. 176 (1968) 581.

[6] Monceau, P., Thèse, Grenoble (1970).

[7] Maki, K., J. Low Temp. Phys. 3 (1970) 545.
[8] Maki, K., Tokyo Summer Lectures on "Physics of Quantum Fluids » (Syokabo, Tokyo) 1971, p. 117.

[9] Saint-James, D., Phys. Lett. 16 (1965) 218.

[10] Yamafugi, K., Kusayanagi, E. et Irie, F., Phys. Lett. 21 (1966) 11

[11] Waysand, G., Thèse Orsay (1972).

[12] Monceau, P., Saint-James, D. et Waysand, G., Proceedings of LT 13, to be published. 